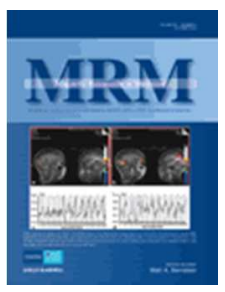

Influence of muscle fibre orientation on water and metabolite relaxation times, magnetization transfer, and visibility in human skeletal muscle

\begin{tabular}{|r|l|}
\hline Journal: & Magnetic Resonance in Medicine \\
\hline Manuscript ID: & MRM-15-15824.R1 \\
\hline Wiley - Manuscript type: & Full Paper \\
\hline Date Submitted by the Author: & n/a \\
\hline Complete List of Authors: & $\begin{array}{l}\text { MacMillan, Erin; University Bern, DRNN / DKF; University and ETH Zurich, } \\
\text { Institute for Biomedical Engineering } \\
\text { Bolliger, Christine; University Bern, DRNN / DKF } \\
\text { Boesch, Chris; University Bern, DRNN / DKF } \\
\text { Kreis, Roland; University Bern, DRNN / DKF }\end{array}$ \\
\hline Research Type: & $\begin{array}{l}\text { Spectroscopy/Spectroscopic Imaging < Technique Development }< \\
\text { Technical Research, Bioeffects < Biophysics < Technical Research }\end{array}$ \\
\hline Research Focus: & $\begin{array}{l}\text { Muscle < Musculoskeletal, Normal < Biochemistry }<\text { Pathology / Other }< \\
\text { Musculoskeletal }\end{array}$ \\
\hline
\end{tabular}




\title{
Influence of muscle fibre orientation on water and metabolite relaxation times, magnetization transfer, and visibility in human skeletal muscle
}

\author{
Erin Leigh MacMillan ${ }^{1,2}$, Christine Sandra Bolliger ${ }^{1}$, Chris Boesch ${ }^{1}$, and Roland Kreis ${ }^{1}$ \\ 1. Department of Clinical Research and Institute of Diagnostic, Interventional and Pediatric Radiology, \\ University of Bern, Bern, Switzerland \\ 2. Institute for Biomedical Engineering, University and ETH Zurich, Zurich, Switzerland
}

Running Head: Magic angle effects for water and metabolites in human muscle

Manuscript Word Count: 3901

Keywords: proton MR spectroscopy; muscle; orientation; magnetization transfer; magic angle;

\section{Corresponding author:}

Prof. Dr. sc. nat. Roland Kreis

University Bern,

Inselspital, P.O. Box 35,

CH-3010 Bern, Switzerland

Tel: +41-31-632 8174, Fax: +41-31-632 0580

Email: roland.kreis@insel.ch 


\section{ABSTRACT}

Purpose: To gain a deeper understanding of the influence of skeletal muscle fibre orientation on metabolite visibility, magnetization transfer from water, and water proton relaxation rates in ${ }^{1} \mathrm{H} M R$ spectra.

Methods: Non-water-suppressed MR spectroscopy was performed in tibialis anterior muscle (TA) of ten healthy adults, with the TA oriented either parallel or at the magic angle to the $3 \mathrm{~T}$ field. Spectra were acquired with metabolite-cycled PRESS, and water inversion from 50 to $2510 \mathrm{~ms}$ prior to excitation. Water proton $T_{2}$ relaxation was sampled with STEAM with echo times from 12 to $272 \mathrm{~ms}$.

Results: Apparent concentrations of total creatine ( $\mathrm{tCr}$ ), taurine, and trimethylammonium compounds were reduced by $29 \%$ to $67 \%$ when TA was parallel to $\mathrm{B}_{0}$. Both $\mathrm{tCr}$ peak areas were strongly correlated to the methylene peak splitting. Magnetization transfer rates from water to $\mathrm{tCr} \mathrm{CH}_{3}$ were not significantly different between orientations. Water $T_{1}$ 's were similar between orientations, but $T_{2}$ 's were statistically significantly shorter by $1 \mathrm{~ms}$ in the parallel orientation $(\mathrm{p}=0.002)$.

Conclusion: Muscle metabolite visibilities in MR spectroscopy and water $\mathrm{T}_{2}$ times depend substantially on muscle fibre orientation relative to $\mathrm{B}_{0}$. In contrast, magnetization transfer rates appear to depend on muscle composition, rather than fiber orientation.

Keywords: MR spectroscopy; skeletal muscle; orientation; magnetization transfer; magic angle; 


\section{INTRODUCTION}

Skeletal muscle metabolism, metabolite compartmentation, and tissue microstructure can be probed non-invasively in vivo using MR spectroscopy. The dense muscle fibre microstructure gives rise to orientation dependent MR features, with anisotropic overall motion of the creatine $(\mathrm{Cr})$ and phosphocreatine ( $\mathrm{PCr}$ ) molecules causing residual dipolar couplings first described for the total observed creatine $(\mathrm{tCr}=\mathrm{Cr}+\mathrm{PCr})$ resonances $(1,2)$ while orientation dependence was later also reported for most of the other muscle metabolites. Taurine, trimethylammonium containing compounds (TMA), and carnosine resonances were all shown to be affected by muscle fibre microstructure since their spectral patterns and/or amplitudes depend on the relative orientation of muscle fibers with respect to the main magnetic field (3-7).

In addition, the exchange of water in the hydration sphere of $\mathrm{tCr}$ molecules with free water can be investigated via an inversion transfer experiment, where the $\mathrm{tCr}$ peak areas attenuate in response to frequency-selective water inversion. Previous investigations of this effect have demonstrated similar attenuation of $\mathrm{tCr}$ peak areas in the human brain (8), skeletal muscle $(8,9)$, as well as in rat brain (10). Recent in vitro ${ }^{1} \mathrm{H}$ NMR magic angle spinning observations suggest that - besides the chemical partitioning into unphosphorylated $\mathrm{Cr}$ and $\mathrm{PCr}$ - there are two pools of $\mathrm{Cr}$; one motionally-free, and one motionally-restricted, where the latter may not be visible in vivo but may be involved in the transfer of magnetization with water (11). Surprisingly, our previous inversion transfer experiment in human calf muscles revealed a significant difference in magnetization transfer (MT) rates between the tibialis anterior (TA) and soleus muscles (9), which was attributed to either varying muscle composition or the differing muscle fibre orientation.

Muscle fibre orientation effects on water proton relaxation times have been investigated early on in ex vivo rat $\mathrm{TA}$, revealing an orientation effect on $\mathrm{T}_{2}$ but not $\mathrm{T}_{1}$ (12). However, the so-called magic angle effect is only well established in vivo for cartilage tissue ((13) and refs. therein), and to date the effects of muscle fibre orientation on water relaxation in human skeletal muscle in vivo has not been characterized.

The present study sought to answer several questions regarding the NMR properties of skeletal muscle water and metabolites in humans: 1) do water-tCr MT rates depend on muscle fibre orientation or composition; 2) can the varying visibility of skeletal muscle metabolites in different fibre orientations be confirmed at $3 \mathrm{~T}$ and using non water suppressed MRS; and 3) do water proton relaxation times change with muscle fibre orientation? 


\section{METHODS}

\section{Subjects}

Ten healthy adults aged 20 to 46 years, (median 26 years, 3 males and 7 females) of varying degrees of fitness and without any history of musculoskeletal disorders were recruited. The study was approved by the local institutional review board, and written, informed consent was obtained.

\section{MRS Data Acquisition}

Subjects were scanned on a 3T Siemens VERIO MR system with the body coil for transmission and a flexible phased array coil for reception. Subjects were positioned lying on their right side on a home built support on top of the scanner table, with their lower right leg through a rotatable coil mount. The support allowed the subject's body to slide along the table when the right leg was bent or straightened, so as to keep the coil at the same position along the external magnetic field. Thus, the entire measurement could be performed twice, once with the lower leg aligned at the magic angle and once parallel with respect to the external magnetic field, without having to adjust the positioning of the coil. However, it should be noted that the flexible coil, which is partly wrapped around the calf, was rotated along with the leg such that the receive performance of the coil was smaller at the bent position than when the leg was parallel to $\mathrm{B}_{0}$ because the main axis of some of the coil elements did not remain perpendicular to $\mathrm{B}_{0}$. $T_{2}$ weighted images were acquired and used to prescribe a spectroscopy voxel (mean volume $6.9 \mathrm{~mL}$ ) in the TA with as little contamination from extramyocellular lipid deposits as possible, as illustrated in Fig. 1. Voxel dimensions and voxel orientiation with respect to the leg were kept the same within a volunteer for the two orientations, with the exception of one case in which the parallel orientation voxel was $5 \%$ smaller.

PRESS localized spectra were acquired without water suppression (WS) by presaturation, but using metabolite cycling $(9,14)$ with TE/TR $=26 / 4000 \mathrm{~ms}, 2$ start-up cycles followed by 32 acquisitions, 2048 samples, and a bandwidth of $4000 \mathrm{~Hz}$. The metabolite cycling pulse had a duration of $22 \mathrm{~ms}$, with a delay of $7 \mathrm{~ms}$ to the middle of the PRESS $90^{\circ}$ pulse. Magnetization transfer between water and $\mathrm{tCr}$ was measured using an inversion transfer experiment in which an additional Gaussian-shaped frequency selective pulse of $12 \mathrm{~ms}$ duration was applied to invert the water resonance with varying delay times (TI) before the PRESS acquisition ( $\mathrm{TI}=50,510,660,810,960,1110,1260,1560,2510 \mathrm{~ms}$ ), in addition to a non-water-inverted (NI) measurement. Individually acquired free induction decays (FID's) were stored separately for later post-processing. The measurement took $128 \mathrm{~s}$ for each TI time. In addition, single shot non-WS STEAM spectra with varying TE times (TE = 12, 14, 17, 20, 24, 29, 34, 40, 48, 68, 96, 136, 192, $272 \mathrm{~ms}$ ) were also acquired. Automatic $\mathrm{B}_{0}$ shimming was performed, and the transmitter power was 
optimized for a $90^{\circ}$ flip angle in the prescribed voxel using the manufacturer's work-in-progress $\mathrm{B}_{1}$ mapping method.

\section{Data Analysis}

Spectra were preprocessed as described previously (9). Briefly, individual NI shots were frequency aligned with the water peak, then eddy-current corrected with its water-only signal. Water inverted shots were frequency aligned using the $3.03 \mathrm{tCr}$ methyl peak, and eddy current correction was performed using the water signal obtained from the TI $=2510$ water signal, then frequency and phase aligned to the NI case. Residual water and signals in the lipid region (0 to $2.7 \mathrm{ppm}$ ) were removed from the metabolite spectra by Hankel-Lanczos Singular Value Decomposition prior to fitting. Both metabolite and water spectra were fit in FiTAID (15), which fits peak areas at all TI times simultaneously. This is illustrated and described in Fig. 1. Base spectra had been set up heuristically, with the tCr spectrum as one doublet for $\mathrm{CH}_{2}$ and one triplet for $\mathrm{CH}_{3}$, a singlet for TMA, and a doublet of doublets for taurine.

The longitudinal magnetizations of water and $\mathrm{tCr}$ were modeled as described previously (9) to extract the water and metabolite $T_{1}$ relaxation times and magnetization transfer rates. In brief, the water inversion recovery curve was initially fit for each volunteer to determine the water $T_{1}$ time, inversion pulse efficiency, and equilibrium magnetization. These parameters were then used as constants in the fit of the inversion transfer curves for each metabolite's peak area. The metabolite curves were modeled on an idealized pulse sequence, considering only the effects of the water inversion pulse and the $90^{\circ}$ pulse in PRESS, and was described using the two-pool Bloch-McConnell equations (16). The metabolite curve fitting optimized each metabolite's equilibrium magnetization, $T_{1}$ in absence of exchange, and forward transfer rate constant from water to metabolites, while the backwards transfer rate constant was set to fulfill steady state conditions. Non-WS STEAM spectra with varying TE times were fit with a single water peak, and peak areas were fit to a mono-exponential decay using least-squares minimization. The Wilcoxon signed-rank test was used to compare $\mathrm{T}_{1}$ times and magnetization transfer rates of the $\mathrm{tCr} \mathrm{CH}_{3}$ peak in between the two fibre orientations.

To determine absolute concentrations for $\mathrm{tCr}-\mathrm{CH}_{2}$ and $-\mathrm{CH}_{3}$, TMA, and taurine, metabolite signals were scaled to the water peak area with the assumption that skeletal muscle is $78 \%$ water (17) and fully visible, independent of fibre orientation. The water peak area was corrected with the measured $T_{1}$ and $T_{2}$ values, and scaled by a factor of 1.25 to account for the effect of direct saturation by the metabolite cycling pulses. The tCr and TMA peak areas were corrected with orientation-independent relaxation values reported previously (18). Both $\mathrm{tCr}$ peak areas were corrected with the same $\mathrm{T}_{2}$ values, and the 
taurine peak area was corrected with the $\mathrm{T}_{2}$ value of $\mathrm{tCr} \mathrm{CH}_{3}$. Metabolite concentrations between the magic angle and parallel orientations were compared using the Wilcoxon signed-rank test. $\mathrm{tCr}$ concentrations for each resonance $\left(\mathrm{CH}_{2}\right.$ and $\left.\mathrm{CH}_{3}\right)$ and $\mathrm{T}_{2}$ values of water were fit to a linear relationship with $\mathrm{CH}_{2}$ peak splitting. Signal pre-processing, model optimization, statistics, and graphing were performed in MATLAB (7.10.0 R2010a, The Mathworks, Natick, MA, USA).

\section{RESULTS}

Creatine peak areas at 3.03 and $3.93 \mathrm{ppm}$ in the tibialis anterior muscle are affected by frequency selective inversion of water, as demonstrated in Fig. 2, with maximal difference from the non-inverted spectrum approximately $1 \mathrm{~s}$ following water inversion. This effect is similar at both the magic angle and parallel orientations with respect to the external magnetic field. Inversion transfer modeling of the change in $\mathrm{tCr} \mathrm{CH}_{3}$ peak area with water inversion delay time is shown in Fig. 3, and revealed MT rates of (median $\left(25^{\text {th }} \& 75^{\text {th }}\right.$ percentiles $\left.)\right) 0.43(0.37-0.48) \mathrm{s}^{-1}$ and $0.41(0.36-0.51) \mathrm{s}^{-1}$ in the tibialis anterior at the magic angle or parallel to the $\mathrm{B}_{0}$ field, respectively. The median longitudinal relaxation times were determined to be $1.14(1.00-1.32) \mathrm{s}$ and $1.47(1.12$ - 1.69) s, respectively. No significant difference between muscle fibre orientations was detected for either the MT rate $(p=0.85)$ or the tCr $\mathrm{CH}_{3} \mathrm{~T}_{1}$ relaxation time $(\mathrm{p}=0.10)$.

All apparent metabolite concentrations were significantly higher in the magic angle orientation as compared to the parallel orientation $(\mathrm{p}<0.01)$, as illustrated in Fig. 4. The median $\mathrm{tCr} \mathrm{CH}_{2}$ concentration exhibited a $33 \%$ decrease from $28.8(27.7-30.5) \mathrm{mM}$ at the magic angle to $19.2(18.7-20.8) \mathrm{mM}$ in the parallel orientation, while the $\mathrm{tCr} \mathrm{CH}_{3}$ concentrations exhibited a $29 \%$ drop from $35.2(34.0-37.2) \mathrm{mM}$ to $25.0(23.3-27.5) \mathrm{mM}$. The TMA and taurine concentrations exhibited even stronger decreases, with TMA decreasing 55\% from $10.4(8.8-13.4) \mathrm{mM}$ to $4.7(3.9-5.2) \mathrm{mM}$, and taurine decreasing $67 \%$ from $15.8(14.3-18.0) \mathrm{mM}$ to $5.2(4.6-6.1) \mathrm{mM}$.

The measured concentrations of both $\mathrm{tCr}$ peaks were strongly correlated to the $\mathrm{CH}_{2}$ doublet splitting of $\mathrm{tCr}\left(\mathrm{R}^{2}=0.79 \mathrm{p}<1 \times 10^{-6}\right.$ for $\mathrm{CH}_{2}$, and $0.65, \mathrm{p}<1 \times 10^{-4}$ for $\left.\mathrm{CH}_{3}\right)$, as shown in Fig. 5. Furthermore, the slope of the linear relationship was similar between the $\mathrm{CH}_{2}$ and $\mathrm{CH}_{3}$ peaks.

The water proton $\mathrm{T}_{1}$ relaxation time was also extracted from the inversion transfer model, and was similar between muscle fibre orientations: $1.34(1.32-1.38) \mathrm{s}$ at the magic angle, and $1.34(1.32-1.35) \mathrm{s}$ for the parallel orientation $(\mathrm{p}=0.97)$. Conversely, the water proton $\mathrm{T}_{2}$ relaxation time determined from the non-WS STEAM TE series was significantly different between orientations: $28.7(28.3-29.9) \mathrm{ms}$ at the magic angle and $27.6(27.2-27.9) \mathrm{ms}$ in the parallel orientation $(\mathrm{p}=0.002)$. Its dependence on orientation, 
represented by the $\mathrm{tCr} \mathrm{CH} 2$ peak splitting, is plotted in Fig. 6, where a strong correlation is observed over the whole cohort $\left(\mathrm{R}^{2}=0.60 \mathrm{p}<0.0001\right)$.

\section{DISCUSSION}

Selective inversion of the water resonance leads to attenuation of the total observed creatine resonances in human tibialis anterior muscle, as determined by ${ }^{1} \mathrm{H}$ MRS without water presaturation. The MT rates reported here are in agreement with our previous study in the same muscle (9), and exhibit a similar time course to earlier reports in human brain and skeletal muscle (8). Our previous work demonstrated a significant difference in water-tCr MT rates between the tibialis anterior and soleus muscles, which could be attributable to either different muscle compositions or fibre orientations. The present study found similar MT rates in the tibialis anterior muscle aligned with the external magnetic field and at the magic angle, suggesting that the varying water-tCr MT rates reported earlier (9) probably arise from different fibre type compositions between the tibialis anterior and soleus muscles, though this will need corroboration with studies including determinations of fiber type.

Histological examination of post-mortem human muscle tissue determined that the tibialis anterior is composed of roughly $78 \%$ type 1 and $22 \%$ type 2 fibres, while the soleus is composed of $96 \%$ type 1 and $4 \%$ type 2 fibres (19). Given that the kinases involved in the creatine-phosphocreatine energy shuttle are bound to the mitochondria and myofibrils (20), and that there is a larger mitochondrial content in type 1 fibres than type 2 fibres, type 1 fibres may have a higher ratio of bound to free creatine, which might enable a faster diffusion of magnetization from water to free creatine via the bound pool. In this case, it would be expected that muscles high in type 1 fibres would experience faster water to tCr MT rates, as was found in our previous study (9).

Indeed, high resolution magic angle spinning (HRMAS) ${ }^{1} \mathrm{H}$ NMR spectroscopy of fresh human skeletal muscle tissue resolved the tCr resonances into 3 separate peaks assigned to $\mathrm{PCr}$, as well as mobile and motionally restricted creatine (11). Given that the intensities of the PCr and creatine multiplets are similar in spectra of spinning and non-spinning samples, the restricted creatine pool is likely not to be observable without magic angle spinning, i.e. in vivo. Moreover, nuclear Overhauser effect spectroscopy of these samples indicated that this motionally restricted creatine pool is confined in a small space with water molecules (11). The confirmed presence of a motionally restricted creatine pool in close association with water molecules invites the speculation that the MT-related attenuation of the $\mathrm{tCr}$ peak areas in the present study occurs due to transfer of the inverted water magnetization to the motionally restricted creatine pool, followed by an exchange between the restricted and free creatine pools to produce the 
observed effect on the $\mathrm{tCr}$ peak areas. Since bound pools were not observed for any other metabolites in HRMAS and the MT is clearly much larger for creatine than other peaks, this link may be more than pure coincidence.

Regarding the observed reduced visibility of the metabolite signals off the magic angle, there are several related findings in the published literature. In terms of the creatine signals, there is an ongoing debate about the full visibility of unphosphorylated creatine. Strong correlations between exerciseinduced depletion of the phosphocreatine signal in ${ }^{31} \mathrm{P}$ MRS and ${ }^{1} \mathrm{H} \mathrm{tCr}$ peak areas in human TA suggested that the entire tCr methylene signal arises from PCr and that free creatine is MR invisible (21). For the methyl peak pattern, this study suggested a partial visibility, with changes in lineshape. This finding was corroborated in a study with electric stimulation of muscle contraction (22). The measurements on the effect of exercise were performed in the parallel orientation, which is now demonstrated to have a $29 \%$ decrease in the $\mathrm{tCr} \mathrm{CH}_{3}$ peak area and a $33 \%$ decrease in $\mathrm{tCr} \mathrm{CH}_{2}$ peak area compared to the magic angle setting. Given that $20-30 \%$ of total creatine is known to be unphosphorylated from chemical biopsy measurements in resting conditions for human skeletal muscle, these two observations would match up well, if one assumes that unphosphorylated creatine becomes visible at the magic angle, while at other angles the dipolar splitting could be substantially larger and/or less uniform for free creatine than for $\mathrm{PCr}$, and thus free creatine signals might be broadened and distributed beyond detectability over a larger frequency range. This explanation is also supported by the determined tissue content for total creatine, which is in taking with the biopsy literature for the magic angle, but not the parallel arrangement. In contrast, the correlation between ${ }^{1} \mathrm{H}$ and ${ }^{31} \mathrm{P} \mathrm{tCr}$ peak areas was not observed in mouse gastrocnemius muscle pre- and post-mortem (though there were some lineshape effects specific to the off-magic angle settings), and furthermore, similar $\mathrm{tCr} \mathrm{CH}_{2}$ visibility was observed for both wild type and creatine kinase deficient mice who exhibit decreased PCr levels (23). However, these measurements were done at $7 \mathrm{~T}$ and in another species, which still leaves room for the above interpretation for $3 \mathrm{~T}$ and human muscle. In addition to, and in support of, the theory that creatine visibility is limited off the magic angle, it has been reported that creatine indeed shows larger dipolar couplings than PCr - at least in model systems featuring residual dipolar couplings $(24,25)$.

The exact nature of the orientation dependence of apparent concentrations or relaxation times cannot be derived from our data as given in Figs 5 and 6. The suggested linear relation is not founded in the data, but is just the most basic correlation. Given that dipolar couplings may play a crucial role for the MR visibilities, either through residual dipolar splittings or through relaxation enhancements, it is reasonable to use the residual dipolar coupling of $\mathrm{tCr}$ as first approximation of an orientation measure, but the relations may equally well be non-linear. 
Further evidence with regard to variable MR-visibilities for metabolite peaks as function of orientation, in particular for TMA and tCr, comes from a spectroscopic imaging study of the human calf muscle. At $1.5 \mathrm{~T}$ the authors demonstrated the variability of $\mathrm{tCr}$ and TMA peak areas across different muscles, which was suggested to partially depend on fibre orientation (3). This finding was further supported by similar spectroscopic imaging of the human calf at $1.5 \mathrm{~T}$ with the lower leg oriented at $0^{\circ}$, $30^{\circ}$, and $70^{\circ}$ with respect to the main magnetic field, revealing nearly twice the tibialis anterior $\mathrm{TMA} / \mathrm{tCr}$ ratio at $70^{\circ}$ versus $0^{\circ}(4)$, which is consistent with our finding of a larger orientation dependence for TMA than $\mathrm{tCr}$. On the other hand, two other investigations did not find an effect of orientation on $\mathrm{tCr}$ peak area, but both studies experienced relevant limitations. Gao et al. did not observe changes in the $\mathrm{tCr} \mathrm{CH}_{3}$ peak areas when the human forearm muscles were placed at different angles to the $\mathrm{B}_{0}$ field, however the $\mathrm{tCr}$ $\mathrm{CH}_{3}$ peak splitting in the parallel orientation was not accounted for in the spectral fitting (26). In addition, $\mathrm{tCr}$ measurements in the human soleus muscle under muscle stretching caused by varying foot orientations did not reveal changing peak areas, however, the induced muscle fibre orientation changes may not have been strong enough to produce an amplitude effect (27).

Furthermore, for TMA the visibility may depend on field strength, too. TMA is a composite peak with choline, phosphocholine, glycerophosphorylcholine, betaine, carnitine, acetylcarnitine, and acylcarnitines all contributing and all with the potential for residual dipolar couplings and differential visibilities. From work at $1.5 \mathrm{~T}$ (28) and 3T (29), it appeared that free carnitine is only partially visible both on and off the magic angle. This was concluded from difference spectra obtained for the exerciseinduced transfer of carnitine to acetylcarnitine, which showed a well resolved positive peak for the appearance of the TMA singlet (3.19 ppm) of acetylcarnitine, but only a broad negative peak for the disappearance of the free carnitine TMA contribution in TA, vastus intermedius, and rectus. Similarly, such difference spectra showed an increased signal for total TMA in soleus proportional to the appearance of acetylcarnitine, as determined from the acetyl peak at $2.1 \mathrm{ppm}$. In soleus at $7 \mathrm{~T}(30)$, such difference spectra feature a clear and equally sized negative contribution of free carnitine, thus suggesting full visibility of this component at $7 \mathrm{~T}-$ at least near the magic angle ${ }^{1}$.

The present study confirmed at $3 \mathrm{~T}$ the observations of the $1.5 \mathrm{~T}$ studies that $\mathrm{tCr} \mathrm{CH}_{3}, \mathrm{tCr} \mathrm{CH}_{2}, \mathrm{TMA}$, and taurine peak areas are increased when the TA is at the magic angle as opposed to parallel to the main magnetic field. Moreover, the $\mathrm{tCr} \mathrm{CH}_{3}$ and $\mathrm{CH}_{2}$ concentrations exhibited a strong linear correlation with the splitting of the $\mathrm{tCr} \mathrm{CH}_{2}$ resonance, reinforcing an earlier correlation of TMA/tCr ratio with $\mathrm{tCr} \mathrm{CH}_{2}$ splitting (4).

\footnotetext{
${ }^{1}$ In ref (30), spectra recorded for an off magic angle orientation in gastrocnemius muscle were mentioned, but the results not included in the report.
} 
It should be noted that the orientation dependence could in principle also arise from inherent technical differences between the setups at the two orientations. For one, the receive coil, which was angulated along with the leg, has a lower sensitivity at MA than PA, but since water, which was used as an internal standard, is subject to the same field differences, no relevant net effect is expected. Secondly, $\mathrm{B}_{0}$ field homogeneity at the ROI is better with the leg stretched along the external field than when it is angulated creating substantial perpendicular air-tissue interface components. However, the median difference in water line with was less than $1 \mathrm{~Hz}$, which is expected to have been compensated by the Voigt line model. In addition, a minor part of the orientation dependence of metabolite peak areas may also have arisen through inaccurate model fitting of the MR spectra, where base spectra had been adjusted heuristically for plausibility and minimal residues. In particular, the taurine peaks may not be modelled properly and some of the differences between the orientations could be attributable to the fact that there is no exact or widely accepted model for the peak pattern of taurine as a function of orientation or extent of residual dipolar coupling. Similarly, the overlapping TMA resonance may suffer indirectly somewhat from this deficit, and in addition some of the constituent molecules contributing to the overall TMA peak may actually also show a dipolar pattern off the magic angle, which was not accountable in this evaluation. Whether strictly speaking the whole apparent reduced visibility of metabolites relates to unaccountably complicated and large dipolar splitting effects for some of the constituents or whether strongly reduced $\mathrm{T}_{2}$ 's play the major role is still up for investigation.

Summarizing, the present study adds to a growing body of evidence supporting the relationship between muscle fibre orientation and measured metabolite peak areas, which is crucial for any quantitative assays of muscle metabolites in health and disease.

Finally, the water proton $\mathrm{T}_{2}$ relaxation time was found to be $1 \mathrm{~ms}$ longer when the muscle fibres were near the magic angle, as compared to the parallel orientation, while the $\mathrm{T}_{1}$ relaxation time was indistinguishable between orientations. The orientation dependence of $T_{2}$ and independence of $T_{1}$ for water protons is reminiscent of the so called magic angle effect reported for water associated with collagen fiber orientation (13,31). It has actually been reported early on for ex vivo rat TA muscle (12), where a longer $\mathrm{T}_{2}$ time was also found at the magic angle (12). This study, as well as ours, suggests that a hydration sphere of actin-myosin filaments exhibits a similar behavior as hydrating layers of collagen fibers and that fast exchange with the bulk of muscle water leads to the same, but largely downscaled, effects as for tendons. Whether this effect may have implications in imaging, as is the case for cartilage, is questionable because of the small extent of the $\mathrm{T}_{2}$ shortening, but not excluded. 
In conclusion, we have determined that our previous observation of differing water to creatine MT rates between the tibialis anterior and soleus muscles arises from differences in muscle composition and not orientation dependence. In contrast, metabolite peak areas are orientation dependent, with more accurate concentrations for $\mathrm{tCr}$, TMA, and taurine determined with the muscle fibre oriented at the magic angle where dipolar coupling is negligible. Finally, a small but significant difference in water proton $\mathrm{T}_{2}$ but not $T_{1}$ relaxation rates was detected between muscle fibre orientations, proving that the magic angle effect also occurs for tissues that are not particularly rich in collagen.

\section{Acknowledgments}

We gratefully acknowledge funding from the Swiss National Science Foundation (32000_135743 and 320030_156952), help from our volunteers, and the organizational expertise of Karin Zwygart. 


\section{REFERENCES}

1. Kreis R, Boesch C. Spatially localized, one- and two-dimensional NMR spectroscopy and in vivo application to human muscle. J Magn Reson Ser B 1996;113:103-118.

2. Kreis R, Koster M, Kamber M, Hoppeler H, Boesch C. Peak assignment in localized 1H MR spectra of human muscle based on oral creatine supplementation. Magn Reson Med 1997;37:159-163.

3. Vermathen P, Boesch C, Kreis R. Mapping fiber orientation in human muscle by proton MR spectroscopic imaging. Magn Reson Med 2003;49:424-432.

4. Hu J, Xia Y, Feng W, Xuan Y, Shen Y, Haacke EM, Jiang Q. Orientational dependence of trimethyl ammonium signal in human muscles by $1 \mathrm{H}$ magnetic resonance spectroscopic imaging. Magn Reson Imaging 2005;23:97-104.

5. Boesch C, Kreis R. Dipolar coupling and ordering effects observed in magnetic resonance spectra of skeletal muscle. NMR Biomed 2001;14:140-148.

6. Schröder L, Schmitz C, Bachert P. Phase modulation in dipolar-coupled A2 spin systems: Effect of maximum state mixing in1H NMR in vivo. J Magn Reson 2004;171:207-212.

7. Asllani I, Shankland E, Pratum T, Kushmerick M. Effects of $\mathrm{pH}$ and molecular charge on dipolar coupling interactions of solutes in skeletal muscle observed by DQF, 1H NMR spectroscopy. J Magn Reson 2003;163:124-132.

8. Kruiskamp MJ, de Graaf RA, van der Grond J, Lamerichs R, Nicolay K. Magnetic coupling between water and creatine protons in human brain and skeletal muscle, as measured using inversion transfer $1 \mathrm{H}-$ MRS. NMR Biomed 2001;14:1-4.

9. MacMillan EL, Boesch C, Kreis R. Magnetization exchange observed in human skeletal muscle by non-water-suppressed proton magnetic resonance spectroscopy. Magn Reson Med 2012;70:916-924.

10. De Graaf RA, van Kranenburg A, Nicolay K. Off-resonance metabolite magnetization transfer measurements on rat brain in situ. Magn Reson Med 1999;41:1136-1144.

11. Chen J-H, Wu Y V, DeCarolis P, O’Connor R, Somberg CJ, Singer S. Resolution of creatine and phosphocreatine $1 \mathrm{H}$ signals in isolated human skeletal muscle using HR-MAS 1H NMR. Magn Reson Med 2008;59:1221-1224.

12. Kasturi SR, Chang DC, Hazlewood CF. Study of anisotropy in nuclear magnetic resonance relaxation times of water protons in skeletal muscle. Biophys J 1980;30:369-381.

13. Bydder M, Rahal A, Fullerton GD, Bydder GM. The magic angle effect: A source of artifact, determinant of image contrast, and technique for imaging. J Magn Reson Imaging 2007;25:290-300.

14. MacMillan EL, Chong DGQ, Dreher W, Henning A, Boesch C, Kreis R. Magnetization exchange with water and T1 relaxation of the downfield resonances in human brain spectra at 3.0 T. Magn Reson Med 2011;65:1239-1246. 
15. Chong DGQ, Kreis R, Bolliger CS, Boesch C, Slotboom J. Two-dimensional linear-combination model fitting of magnetic resonance spectra to define the macromolecule baseline using FiTAID, a Fitting Tool for Arrays of Interrelated Datasets. Magn Reson Mater Phy 2011;24:147-164.

16. McConnell HM. Reaction rates by nuclear magnetic resonance. J Chem Phys 1958;28:430-431.

17. De Graaf RA. In Vivo NMR Spectroscopy: Principles and Techniques. 2nd ed. Mississagua: John Wiley \& Sons Ltd.; 2007.

18. Krššák M, Roden M, Mlynárik V, Meyerspeer M, Moser E. 1H NMR relaxation times of skeletal muscle metabolites at 3 T. Magn Reson Mater Phy 2004;16:155-159.

19. Dahmane R, Djordjevic S, Simunic B, Valencic V. Spatial fiber type distribution in normal human muscle Histochemical and tensiomyographical evaluation. J Biomech 2005;38:2451-2459.

20. Bessman S, Carpenter C. The creatine-creatine phosphate energy shuttle. Annu Rev Biochem $1985 ; 54: 831-862$.

21. Kreis R, Jung B, Slotboom J, Felblinger J, Boesch C. Effect of exercise on the creatine resonances in 1H MR spectra of human skeletal muscle. J Magn Reson 1999;137:350-357.

22. Nirkko AC, Rösler KM, Slotboom J. Muscle metabolites: functional MR spectroscopy during exercise imposed by tetanic electrical nerve stimulation. Radiology 2006;241:235-242.

23. In 't Zandt HJ, Klomp DW, Oerlemans F, Wieringa B, Hilbers CW, Heerschap a. Proton MR spectroscopy of wild-type and creatine kinase deficient mouse skeletal muscle: dipole-dipole coupling effects and post-mortem changes. Magn Reson Med 2000;43:517-524.

24. Vilder SJ De, Kruiskamp MJ, Wechselberger R, Czisch M, Nicolay K. Magnetically-oriented bicelles as an in vitro tool to investigate the residual dipolar coupling of creatine and phosphocreatine protons in skeletal muscle. In: Proceedings of the 10th Annual Meeting of ISMRM, Denver, CO, USA, 2000. p. 1007.

25. Agarwal N, Pagès G, D' Silva L, Said N, Kuchel PW, Velan SS. Influence of foot orientation on the appearance and quantification of $1 \mathrm{H}$ magnetic resonance muscle spectra obtained from the soleus and the vastus lateralis. Magn Reson Imaging 2014;32:379-384.

26. Gao F, Bottomley P a, Arnold C, Weiss RG. The effect of orientation on quantification of muscle creatine by 1H MR spectroscopy. Magn Reson Imaging 2003;21:561-566.

27. Marjańska M, Eberly LE, Adriany G, Verdoliva SN, Garwood M, Chow L. Influence of foot orientation on the appearance and quantification of $1 \mathrm{H}$ magnetic resonance muscle spectra obtained from the soleus and the vastus lateralis. Magn Reson Med 2012;68:1731-1737.

28. Kreis R, Jung B, Rotman S, Slotboom J, Boesch C. Non-invasive observation of acetyl-group buffering by 1H-MR spectroscopy in exercising human muscle. NMR Biomed 1999;12:471-476.

29. Boss A, Kreis R, Saillen P, Zehnder M, Boesch C, Vermathen P. Skeletal muscle 1H MRSI before and after prolonged exercise. II. visibility of free carnitine. Magn Reson Med 2012;68:1368-1375. 
30. Ren J, Lakoski S, Haller RG, Sherry AD, Malloy CR. Dynamic monitoring of carnitine and acetylcarnitine in the trimethylamine signal after exercise in human skeletal muscle by 7T 1H-MRS. Magn Reson Med 2013;69:7-17.

31. Peto S, Gillis P. Fiber-to-field angle dependence of proton nuclear magnetic relaxation in collagen. Magn Reson Imaging 1990;8:705-712. 


\section{Figure Captions}

Figure 1. Tibialis anterior muscle spectra obtained with fibres oriented either parallel (upper chart) or at the magic angle to the external magnetic field (lower chart). Top black spectra for each orientation depict the measured spectra averaged over 10 volunteers, coloured lines show the fitted metabolite spectra determined by FiTAID (10) and lower black lines show the residuals. Inset: position of the spectroscopy voxel. FiTAID modeled spectra with Voigt line patterns for a baseline, total creatine (tCr) $\mathrm{CH}_{2}$ and $\mathrm{CH}_{3}$, taurine, and trimethylammonium (TMA) groups. Peak frequencies, phases, and widths were optimized simultaneously for all spectra in the magnetization transfer measurement in each volunteer, while peak areas were permitted to change with water inversion delay time.

Figure 2. Average spectra from the water inversion recovery measurement. Top line shows the nonwater-inverted (NI) spectra obtained parallel or at the magic angle to the external magnetic field, while each subsequent line shows the difference between spectra obtained with increasing water inversion delay times and the NI spectrum. Delay times are given in ms at the right of the figure.

Figure 3. Creatine $\mathrm{CH}_{3}$ peak areas with increasing water inversion delay times or not water inverted (NI). Symbols mark peak areas fit in FiTAID, with error bars showing the Cramér-Rao lower bounds of the peak area fit, and lines demonstrating the fit of the inversion transfer exchange model, which

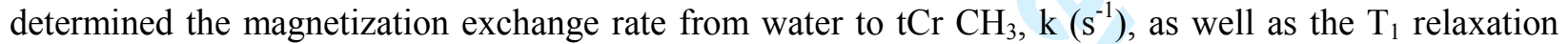
time (s). Medians $\left(25^{\text {th }}\right.$ and $75^{\text {th }}$ quartiles) of the exchange rate and $\mathrm{T}_{1}$ relaxation times for both fibre orientations are given, but neither were significantly different between fibre orientations. The median root mean square errors of the inversion transfer fit expressed as a percent of the non-water-inverted peak area were $8 \%(5-12 \%)$ for the magic angle, and $4 \%(3-7 \%)$ in the parallel orientation.

Figure 4. Comparison of apparent metabolite concentrations between the magic angle (MA) and parallel (PA) orientations. The measured concentration for each metabolite was significantly higher in the magic angle (MA) orientation versus the parallel (PA) orientation $(\mathrm{p}<0.01)$. (Tau: taurine) 
Figure 5. Relationship between muscle fibre orientation, represented by the splitting of the $\mathrm{tCr} \mathrm{CH}_{2}$ doublet, and apparent $\mathrm{tCr}$ peak concentrations. Top: $\mathrm{tCr} \mathrm{CH}_{2}$ concentration versus $\mathrm{tCr} \mathrm{CH}_{2}$ doublet splitting. Bottom: $\mathrm{CH}_{3}$ concentration versus $\mathrm{tCr} \mathrm{CH}_{2}$ doublet splitting. The apparent concentration of each $\mathrm{tCr}$ resonance correlated with the $\mathrm{CH}_{2}$ doublet splitting $\left(\mathrm{p}<10^{-4}\right)$. Diamonds represent measurements intended to be obtained at the magic angle, while circles represent measurements intended to be recorded near a parallel configuration. Standard deviations of the concentration values were always smaller than the symbol size and thus are not shown for clarity. (For abbreviations, see Fig. 4.)

Figure 6. Relationship between muscle fibre orientation, represented by the splitting of the $\mathrm{tCr} \mathrm{CH}_{2}$ doublet, and water $\mathrm{T}_{2}$ relaxation time. (For abbreviations, see Fig. 4.) 

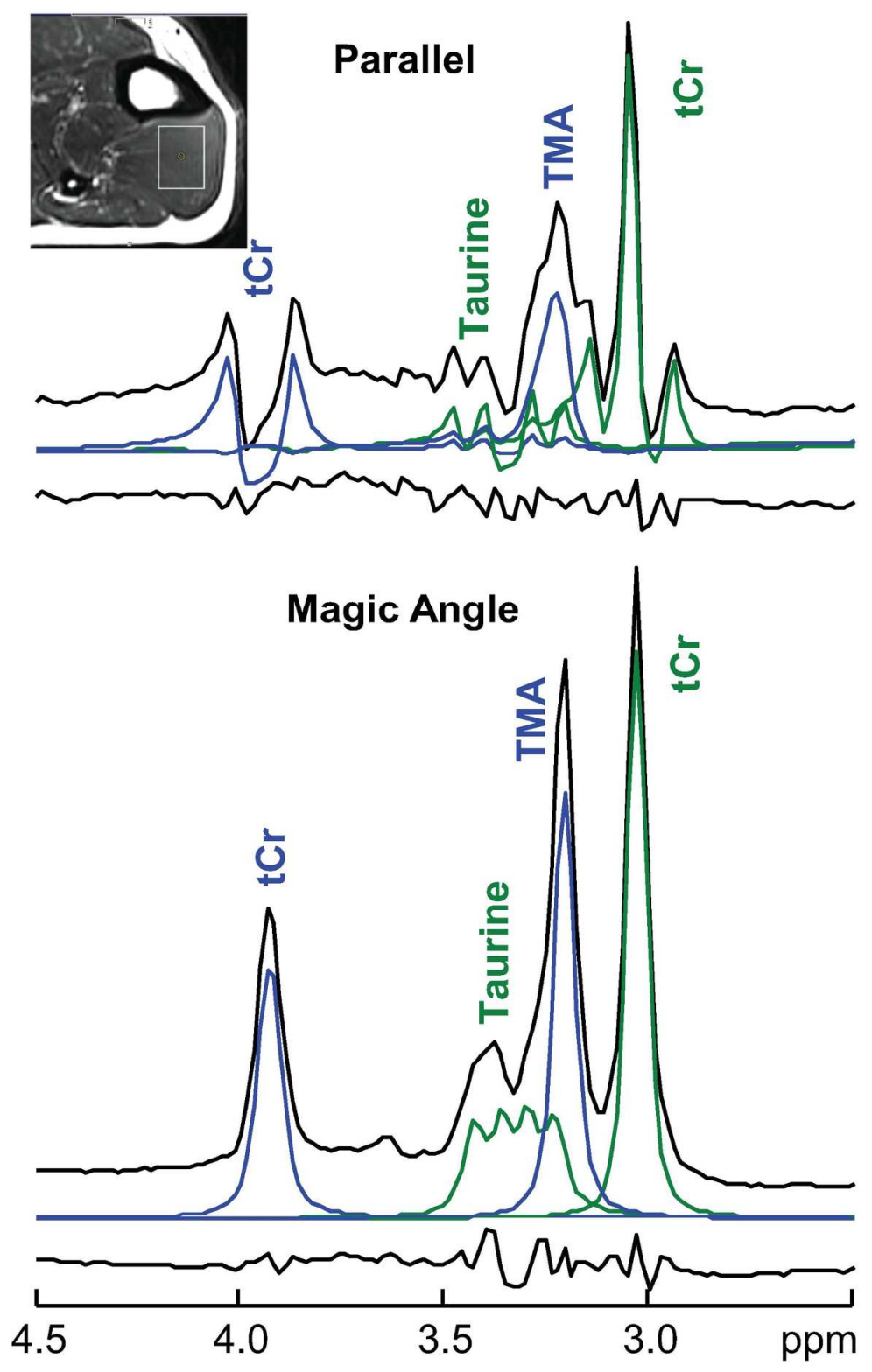

Figure 1.

Tibialis anterior muscle spectra obtained with fibres oriented either parallel (upper chart) or at the magic angle to the external magnetic field (lower chart). Top black spectra for each orientation depict the measured spectra averaged over 10 volunteers, coloured lines show the fitted metabolite spectra determined by FiTAID (10) and lower black lines show the residuals. Inset: position of the spectroscopy voxel. FiTAID modeled spectra with Voigt line patterns for a baseline, total creatine (tCr) $\mathrm{CH} 2$ and $\mathrm{CH} 3$, taurine, and trimethylammonium (TMA) groups. Peak frequencies, phases, and widths were optimized simultaneously for all spectra in the magnetization transfer measurement in each volunteer, while peak areas were permitted to change with water inversion delay time. $132 \times 214 \mathrm{~mm}(300 \times 300$ DPI $)$ 


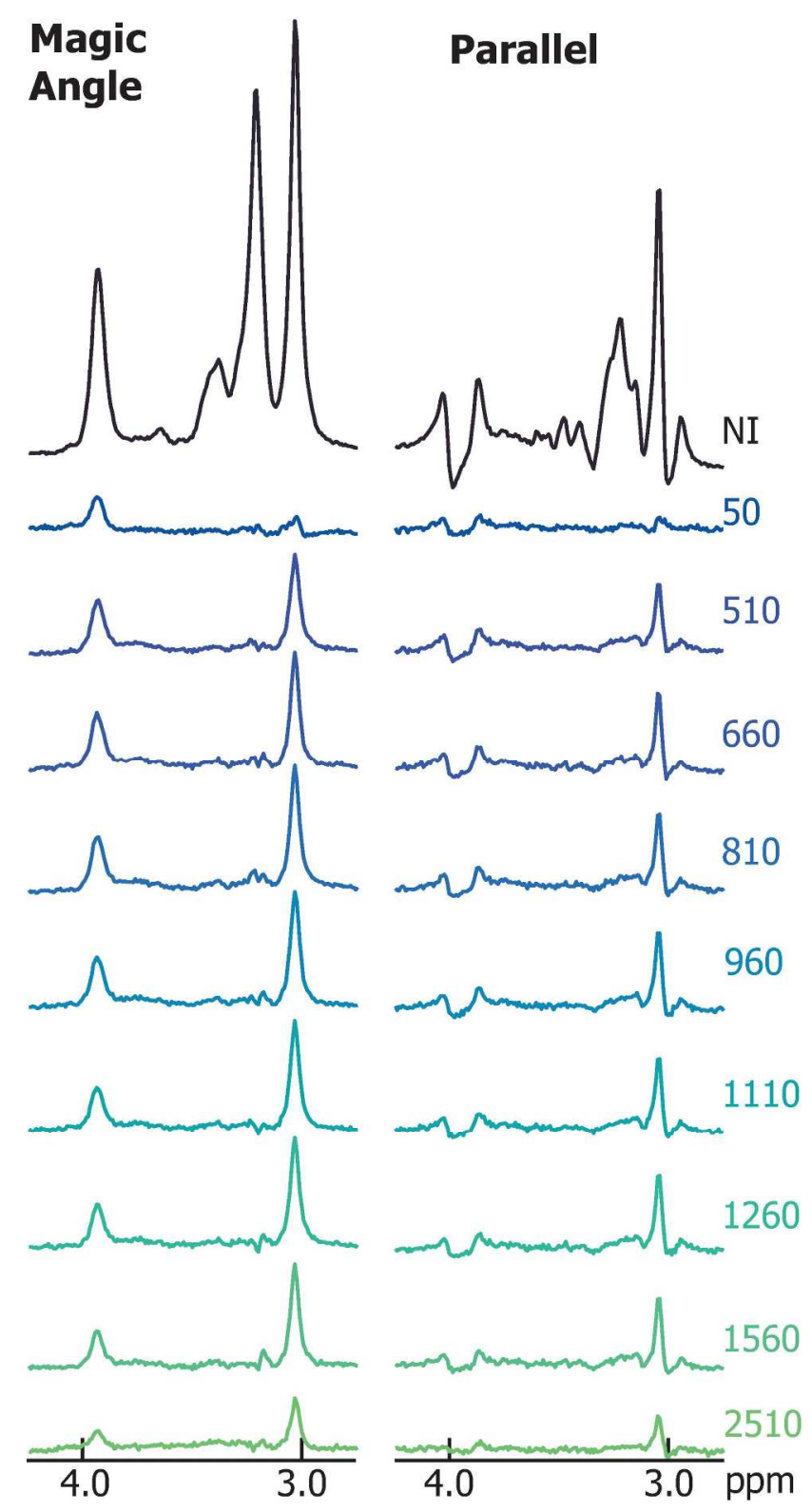

Figure 2.

Average spectra from the water inversion recovery measurement. Top line shows the non-water-inverted (NI) spectra obtained parallel or at the magic angle to the external magnetic field, while each subsequent line shows the difference between spectra obtained with increasing water inversion delay times and the NI spectrum. Delay times are given in $\mathrm{ms}$ at the right of the figure. $158 \times 303 \mathrm{~mm}(300 \times 300$ DPI) 


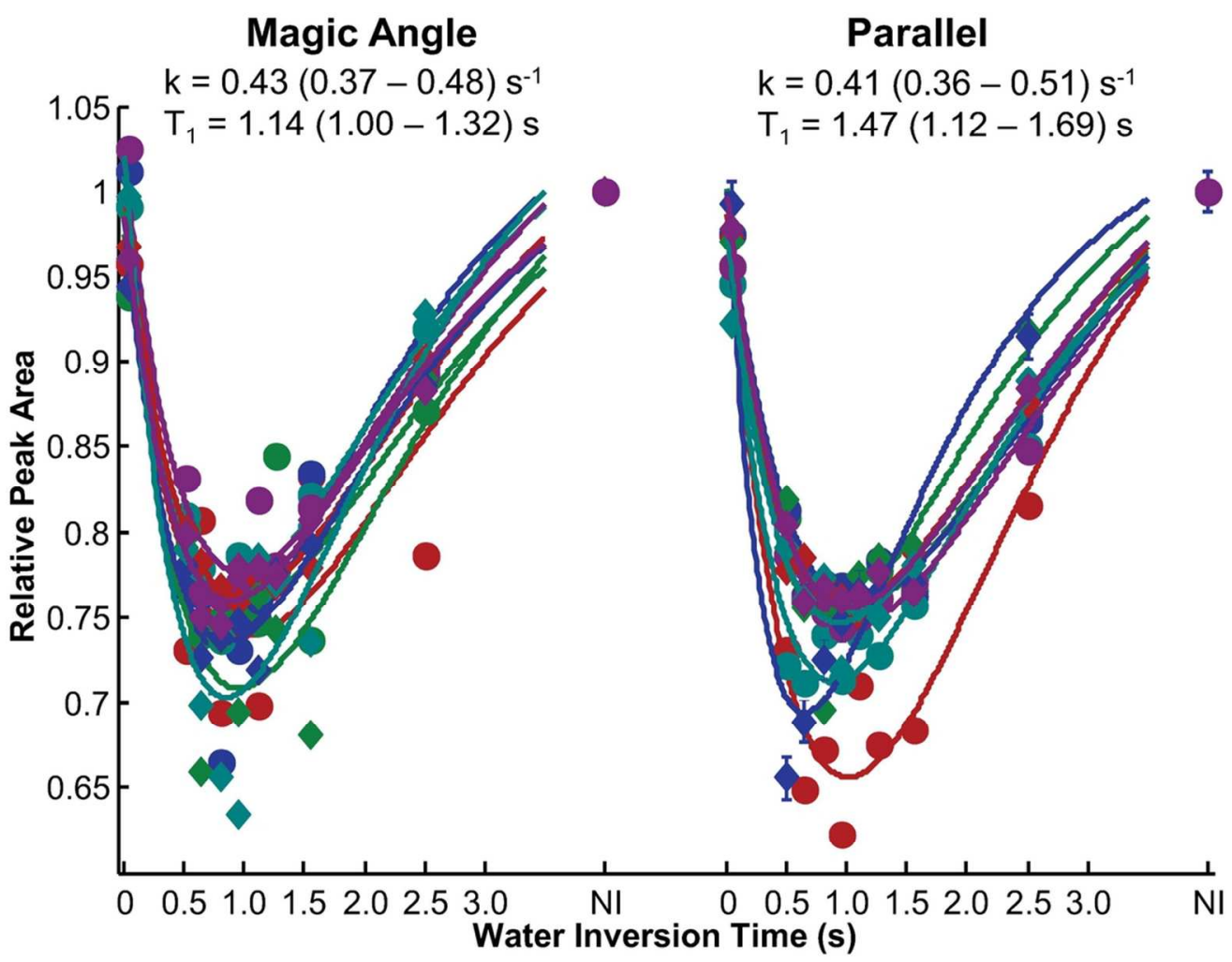

Figure 3. Creatine $\mathrm{CH} 3$ peak areas with increasing water inversion delay times or not water inverted (NI). Symbols mark peak areas fit in FiTAID, with error bars showing the Cramér-Rao lower bounds of the peak area fit, and lines demonstrating the fit of the inversion transfer exchange model, which determined the magnetization exchange rate from water to $\mathrm{tCr} \mathrm{CH} 3, \mathrm{k}(\mathrm{s}-1)$, as well as the T1 relaxation time (s). Medians (25th and 75th quartiles) of the exchange rate and T1 relaxation times for both fibre orientations are given, but neither were significantly different between fibre orientations. The median root mean square errors of the inversion transfer fit expressed as a percent of the non-water-inverted peak area were $8 \%(5-12 \%)$ for the magic angle, and $4 \%(3-7 \%)$ in the parallel orientation. $100 \times 77 \mathrm{~mm}(300 \times 300 \mathrm{DPI})$ 


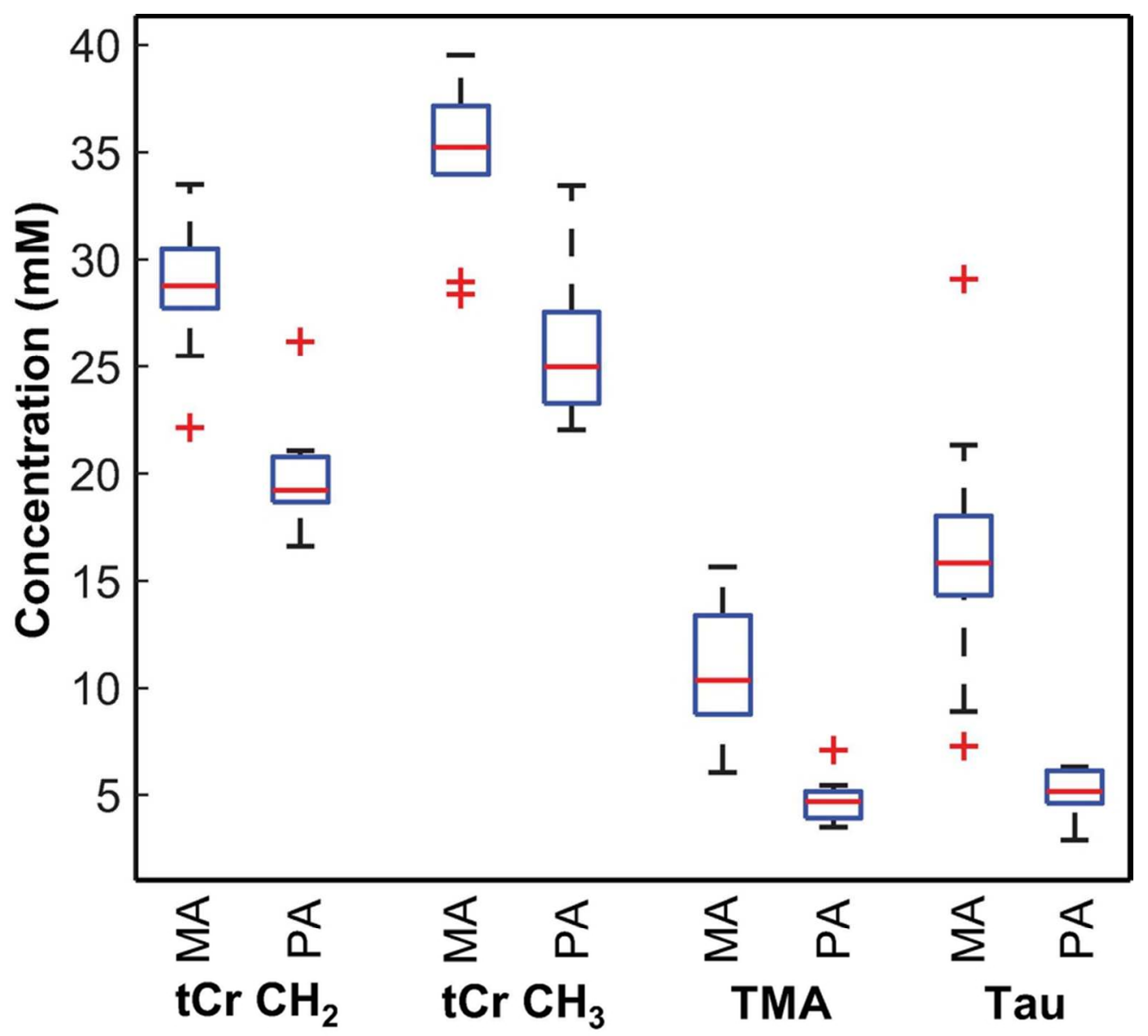

Figure 4.

Comparison of apparent metabolite concentrations between the magic angle (MA) and parallel (PA) orientations. The measured concentration for each metabolite was significantly higher in the magic angle (MA) orientation versus the parallel (PA) orientation $(p<0.01)$. (Tau: taurine) $77 \times 71 \mathrm{~mm}(300 \times 300 \mathrm{DPI})$ 

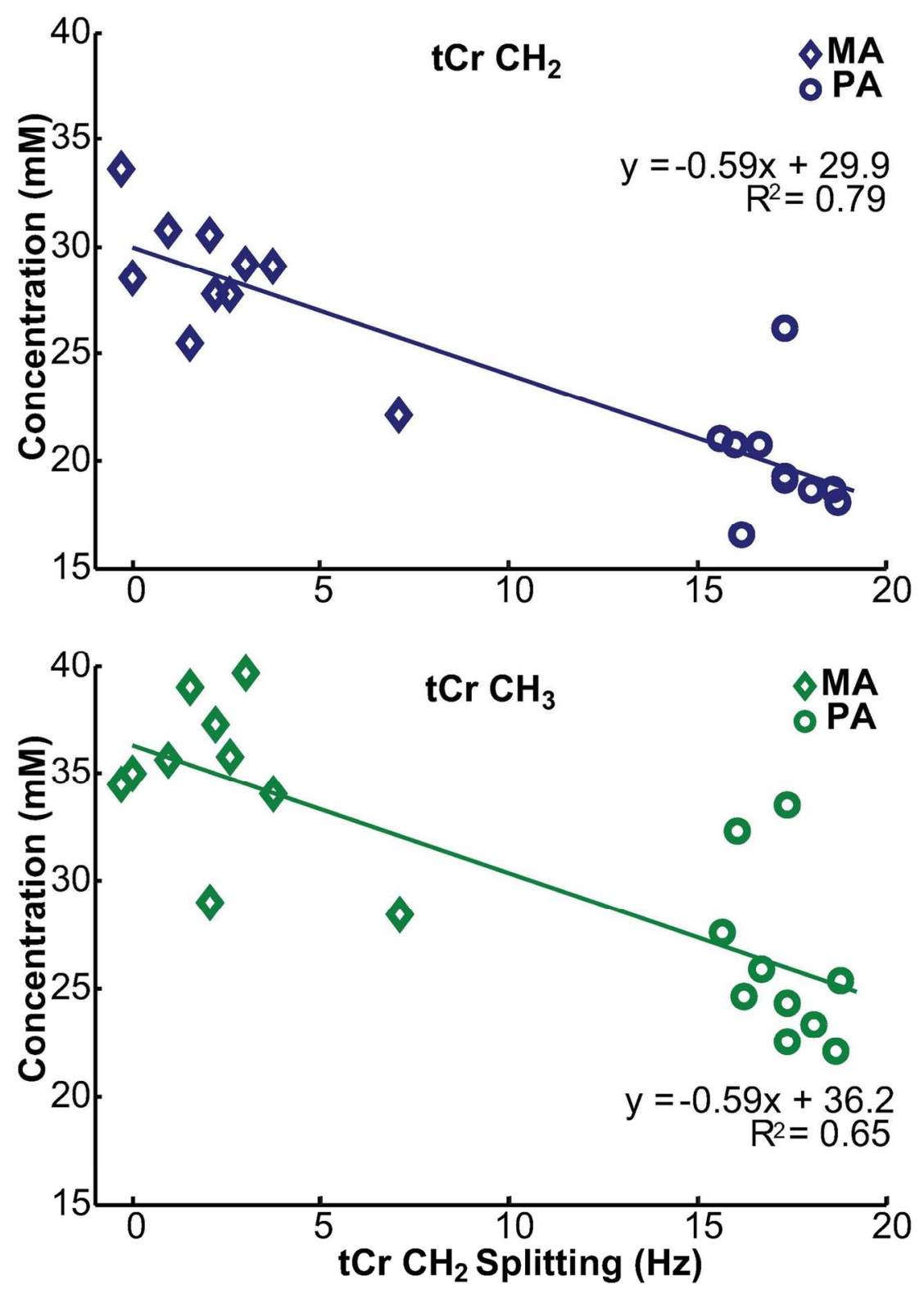

Figure 5.

Relationship between muscle fibre orientation, represented by the splitting of the $\mathrm{tCr} \mathrm{CH} 2$ doublet, and apparent $\mathrm{tCr}$ peak concentrations. Top: $\mathrm{tCr} \mathrm{CH} 2$ concentration versus $\mathrm{tCr} \mathrm{CH} 2$ doublet splitting. Bottom: $\mathrm{CH} 3$ concentration versus $\mathrm{tCr} \mathrm{CH} 2$ doublet splitting. The apparent concentration of each $\mathrm{tCr}$ resonance correlated with the $\mathrm{CH} 2$ doublet splitting $(\mathrm{p}<10-4)$. Diamonds represent measurements intended to be obtained at the magic angle, while circles represent measurements intended to be recorded near a parallel configuration. Standard deviations of the concentration values were always smaller than the symbol size and thus are not shown for clarity. (For abbreviations, see Fig. 4.) $122 \times 174 \mathrm{~mm}(300 \times 300$ DPI) 




Figure 6.

Relationship between muscle fibre orientation, represented by the splitting of the $\mathrm{tCr} \mathrm{CH} 2$ doublet, and water $\mathrm{T} 2$ relaxation time. (For abbreviations, see Fig. 4.) $65 \times 50 \mathrm{~mm}(300 \times 300 \mathrm{DPI})$ 